\title{
Peclet number dependent superdiffusive hydrodynamic dispersion in a site percolation porous media measured by NMR
}

\section{Authors: Joseph D. Seymour, Sarah L. Codd, \& Rainer Kimmich}

NOTICE: this is the author's version of a work that was accepted for publication in Microporous and Mesoporous Materials. Changes resulting from the publishing process, such as peer review, editing, corrections, structural formatting, and other quality control mechanisms may not be reflected in this document. Changes may have been made to this work since it was submitted for publication. A definitive version was subsequently published in Microporous and Mesoporous Materials, [Vol. 29, Issue 1, April 2017] DOI\#10.1016/j.micromeso.2017.04.057"

Seymour, Joseph D., Sarah L. Codd, and Rainer Kimmich. "Peclet Number Dependent Superdiffusive Hydrodynamic Dispersion in a Site Percolation Porous Media Measured by NMR." Microporous and Mesoporous Materials (April 2017). 


\title{
Peclet number dependent superdiffusive hydrodynamic dispersion in a site percolation porous media measured by NMR
}

\author{
Joseph D. Seymour, Sarah L. Codd, Rainer Kimmich
}

\begin{abstract}
The displacement time dependent hydrodynamic dispersion in a model 2D site percolation structure is measured using PGSE NMR. The data indicate superdiffusive scaling of the mean squared displacement at high Peclet numbers, where advective transport dominates, consistent with classic percolation scaling concepts. The time scaling of the mean squared displacement is shown to vary with the Peclet number demonstrating a dependence on the changing dynamics.
\end{abstract}

Anomalous diffusive transport is of significant interest due to the diverse range of phenomena in which it is observed and the role it plays in statistical mechanics models [1,2]. Experimental and theoretical observations of subdiffusion have been reported for a range of phenomena such as charge carrier transport [3], diffusion on percolating clusters [4] and transport in random fracture net-works [5]. In contrast, while superdiffusive processes have been demonstrated for systems exhibiting chaotic dynamics [6], random variation in velocity [7] and turbulence [8], they have been observed experimentally far less with the few examples being Josephson junctions [6] and turbulent flows. Percolation structure porous media are excellent models for environmental transport in fractured rock [9], biomedical transport in gels and tissue [10] and partially saturated porous media [11]. This work reports pulsed gradient spin echo (PGSE) nuclear magnetic resonance (NMR) measurement of superdiffusive hydrodynamic dispersion in a model 2D site percolation system. The data indicates that hydrodynamic dispersion in a 2D site percolation model exhibits a dy-namic dependence on the mean squared

displacement time dependent scaling exponent. This result is in agreement with nu-merical simulations [12] and in contrast to purely geometric ar-guments which predict a constant scaling exponent [9].

Transport phenomena due to combined advection and diffusion plays an elemental role in modeling of transport in microfluidic [13] and porous systems and is known as hydrodynamic dispersion. Experimental and theoretical studies of the mixing of a solute injected in a capillary flow established that the averaged transport behavior followed a macroscopic scale Fickian advection-diffusion, or dispersion, equation (ADE) [14]. The effective Fickian diffusion coefficient being given by $\mathrm{K}^{1 / 4} \mathrm{Dm}_{\mathrm{m}} \mathrm{V}^{2} \mathrm{a}^{2} / 48 \mathrm{Dm}$, for mean velocity $\mathrm{V}$ in a tube of radius a and fluid molecular diffusion $\mathrm{D}_{\mathrm{m}}$, known as the Taylor-Aris dispersion coefficient [15]. This macrotransport approach has been extended to multiphase systems such as porous media and solid liquid suspensions [16], and is important in ap-plications such as contaminant transport and oil recovery in earth formations, industrial reactor design and separation processes like chromatography. The direct connection of this modeling approach and stochastic processes and kinetic theory is well established [17]. Flow, or advection, in porous media results in velocity fluctuations due to the presence of the solid matrix. When the solid matrix exhibits correlated permeability fluctuations [18] or fractal struc-ture [9] the transport is non Fickian or scale dependent. PGSE NMR measurements have been used to characterize the preasymptotic behavior of TaylorAris dispersion [19] and provides 
direct measurement of the displacement length and time dependent hydrodynamic dispersion for flow through porous media [20-23]. Application to model percolation structures has provided velocity [24,25] and molecular diffusion [26] scaling data [27]. Experiments similar to those presented here have analyzed hydrodynamic dispersion time and Peclet number scaling in porous glasses [28]. The paper begins with a theory section 2 . discussing the scaling of hydrodynamic dispersion in percolation structures [9] and simulations [12] related to interpretation of our data. Section 3. provides the experimental details for the model percolation system and the NMR experiments. The data is presented and discussed in the context of current theory and simulations for transport in percolation structures in Sec. 4. Conclusions resulting from this work are provided in Sec. 5.

\section{Theory}

\subsection{Transport}

Hydrodynamic dispersion due to flow in percolation structure porous media is predicted to be governed by the geometric scaling laws of percolation structures [9,29]. The regime of interest is the large Peclet number $(\mathrm{Pe})$ limit where advection is predominant. Following Sahimi [9] we define a grain size Peclet number $P e=\frac{V d_{g}}{D_{m}}$, where $V$ is the mean velocity along the backbone of the percolation network, $d_{\mathrm{g}}$ is the grain, or percolation lattice site scale, $D_{\mathrm{m}}$ the molecular diffusion coefficient of the liquid solute. Three regimes of transport are identified, the low Pe regime in which molecular diffusion dominates, dispersion only along the backbone of the infinite cluster at relatively large $P e$ and a holdup dispersion regime dominated by diffusion from dead end pores to the flow backbone. The regime of interest in this work is that dominated by flow along the backbone. In this regime for site percolation structures the longitudinal, flow direction, mean squared displacement due to dispersion is predicted to scale as $\left\langle Z^{2}(t)>\propto t^{2 /\left(1+\theta_{\mathrm{B}}\right.}\right.$ where $\theta_{\mathrm{B}}=(e-$ $\left.\beta_{\mathrm{B}}\right) / \nu_{\mathrm{p}}$ [9]. The longitudinal dispersion scaling exponent depends on the scaling exponents $e, \beta_{\mathrm{B}}$, and $\nu_{\mathrm{p}}$ for the hydrodynamic permeability $K \sim\left(p-p_{c}\right)^{e}$, the backbone fraction of sites which participate in the flow $X_{\mathrm{B}} \sim\left(p-p_{\mathrm{c}}\right)^{\beta \mathrm{B}}$ and the correlation length $\zeta_{\mathrm{p}} \sim\left|p-p_{\mathrm{c}}\right|^{\nu \mathrm{p}}$ [9]. These classic percolation scalings are valid for site fluid occupation probabilities $p$ near the critical probability for an infinite network, the percolation threshold $p_{c}$. A primary feature of the scaling of the hydrodynamic dispersion from this development is the independence of $2 /\left(1+\theta_{\mathrm{B}}\right)$ from Pe. Using the values of $e, \beta_{\mathrm{B}}$, and $\nu_{\mathrm{p}}$ for 2D site percolation structures superdiffusive transport $<Z^{2}(t)$ $>\sim t^{1.26}$ is predicted [9].

Simulations of flow in correlated percolation structures by Makse et al. [12] indicate a mean transit time which scales as $<t>\sim Z^{\alpha(P e)}$, where the scaling $\alpha(P e)$ is a function of $P e$. The scaling is shown to transition from the expected subdiffusive $\alpha=d_{\mathrm{w}}=2.41$ at low $P e=5 \times 10^{-4}$ to superdiffusive $\alpha=d_{\min }=1.025$ at the highest $P e=1.4$ studied [12]. Here, $d_{\mathrm{w}}$ is the random walk fractal dimension [26] and $d_{\min }$ the fractal dimension of the minimum path distance between two points [30]. It is important to note that the simulations calculate the mean transit time from particles that are traversing the percolation structure along the flow pathlines and hence represents backbone flow statistics [12]. The values of $d_{\mathrm{w}}$ and $d_{\text {min }}$ above are for a correlated percolation structure, whereas for the uncorrelated percolation structure studied here $d_{\mathrm{w}}=2.87$ [12] and $d_{\min }=1.135$ [30], which also predicts a sub to super diffusive transition. Using the relation between the tracer particle probability $P(Z, t)$ that a particle initially at $z_{0}$ has a displacement $Z=Z-$ $z_{0}$ at time $t$ and the first passage time $F(t)$, the equivalence between the scaling of the mean transit time $<t>\sim Z^{\alpha(P e)}$ and the mean squared displacement $\left\langle Z^{2}(t)>\sim t^{2 / \alpha(P e)}\right.$ can be established [9]. Thus for the correlated percolation structure at high $P e$ the simulations of Makse et al. predict near ballistic superdiffusive behavior $<Z^{2}(t)$ $>\sim t^{2 / d \min }=t^{1.95}[12]$. For the site percolation structure in this work $<Z^{2}(t)>\sim t^{2 / d \min }=t^{1.76}$ is also superdiffusive, with larger scaling exponent than the $\left\langle Z^{2}(t)\right\rangle \sim t^{1.26}$ predicted from the classical percolation theory [9].

\subsection{PGSE NMR}

The measured spin echo signal in a PGSE experiment normalized to eliminate magnetic relaxation effects is

$$
E(q, \Delta)=\frac{S(q, \Delta)}{S(q=0, \Delta)}=\int P(Z, \Delta) \exp [i 2 \pi q Z] d Z
$$

where the reciprocal displacement space wave vector $q=(2 \pi)^{-1}$ $\gamma g \delta$ is defined by the experimental pulsed magnetic field gradients of duration $\delta$ and amplitude $g . P(Z, \Delta)$ is the propagator, averaged over the initial spin distribution, for a spin to displace $Z=z-z_{0}$ in the time $\Delta$ between the gradient pulses. The classic means of analyzing molecular diffusion is to plot $\ln (|E|)$ versus $4 \pi^{2} q^{2}(\Delta-\delta / 3)$, which is linear for Gaussian $P(Z, \Delta)$ with slope given by the molecular diffusion [31]. This linear Gaussian decay is in contrast to the behavior in the presence of a complicated flow field as encountered in flow through a porous media. In this more complex case we first look to the initial rapid decay in the low- $q$ limit which provides the process variance or mean squared displacement even for non Gaussian propagators [32], as is seen from a Taylor series expansion of Eqn. (1) $|E|(q, \Delta)=1-4 \pi^{2} q^{2}\left\langle Z^{2}(\Delta)\right\rangle+\cdots$. The fit to this low- $q$ echo attenuation as a function of the observation time $\Delta$ provides the time dependent variance of the dynamics $\left\langle Z^{2}(t=\Delta)>[33,34]\right.$. The ability of PGSE NMR to measure the displacement length scale $1 / q$ and time $\Delta$ dependence provides the means to test the scaling of $\left\langle Z^{2}(t)\right\rangle$. Limiting analysis to the low $q$ regime provides a displacement dynamics wavelength filter. This is demonstrated in discussion of the data and provides the means to selectively measure the hydrodynamic dispersion dynamics from flow along the backbone.

\section{Experimental}

\subsection{Model percolation system}

The model system studied is a quasi 2D site percolation structure near the percolation threshold with $p-p_{\mathrm{c}}=0.03$. The sample construction methodology has been previously reported and used to study the diffusive regime $P e$ « [26], and the mean velocity scaling at higher $P e$ in the advection dominated regime [24,25,27]. The sample consists of seven aligned stacked 2D models in order to increase signal to noise in the experiment and provide average dynamics over an ensemble of systems. The sample structure can be seen in Fig. 1. The grain size is $d_{\mathrm{g}}=300 \mu \mathrm{m}$ and the sample width is $W=60 \mathrm{~mm}$ and length is $L=120 \mathrm{~mm}$. The correlation length is $\zeta_{\mathrm{p}}=\left|p-p_{\mathrm{c}}\right|^{\nu \mathrm{p}}=107.3 d_{\mathrm{g}}=32.2 \mathrm{~mm}$ for $\nu_{\mathrm{p}}=4 / 3$ in $2 \mathrm{D}$, and the sample length $L>3 \zeta_{\mathrm{p}}$. The grain scale $P e$ indicated above is used to characterize the transport, analogous to the definition of Makse et al., and is varied from $P e=0$ to 1130 by varying the volumetric flow rate through the sample [12].

\subsection{PGSE NMR}

The flow compensated double PGSE NMR method we apply involves two pairs of magnetic field gradient pulses configured to refocus coherent motion $[32,33,35]$. This experiment removes the 


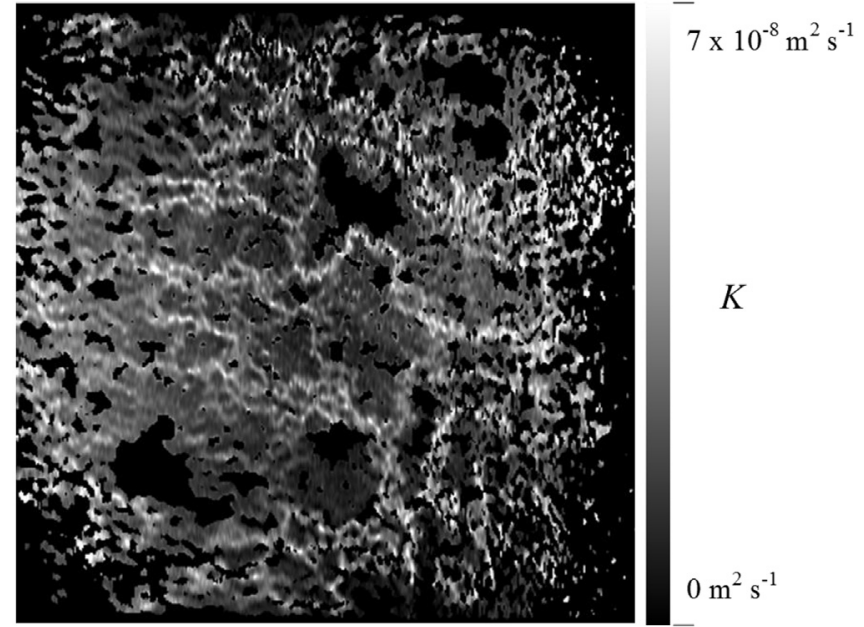

Fig. 1. NMR image of the longitudinal dispersion field in a percolation structure porous media with flow from left to right and $P e=659$. The image has 256 greyscales corresponding to dispersion coefficients from 0 to $7 \times 10^{-8} \mathrm{~m}^{2} \mathrm{~s}^{-1}$. The regions of high dispersivity (white) correspond to the backbone as determined from the spatial distribution of the mean velocity measured by NMR. The spatial resolution is $254 \mu \mathrm{m} /$ pixel and the displacement observation time $\Delta=25 \mathrm{~ms}$.

effect of constant advective transport over the displacement measurement time $2 \Delta$. The displacement measurement time is varied from $2 \Delta=40$ to $840 \mathrm{~ms}$. A $200 \mathrm{MHz}$ horizontal $300 \mathrm{~mm}$ bore NMR system with maximum pulsed magnetic field gradient amplitude $g=0.05 \mathrm{~T} / \mathrm{m}$ and duration $\delta=2 \mathrm{~ms}$ is used with a birdcage rf coil.

\section{Discussion}

Characterization of average velocity behavior for this and other percolation structure porous media by NMR imaging of the spatial velocity distribution along the backbone have been previously presented [24,25]. Fig. 1 shows the spatial distribution of the effective diffusion coefficient in each pixel obtained from a PGSE imaging experiment during flow at $P e=659$. The image clearly demonstrates that hydrodynamic dispersion of the order of $7 \times 10^{-8} \mathrm{~m}^{2} / \mathrm{s}$ occurs along the backbone flow, while diffusive motions of the order $2 \times 10^{-9} \mathrm{~m}^{2} / \mathrm{s}$ are present in dead end pores.

Non spatially resolved PGSE data provides the effective diffusion coefficient for the entire sample. In the case of no flow, i.e. $P e=0$, the dynamics are purely due to molecular diffusion and the spin echo decay data plotted on a Stejskal-Tanner plot is linear in $q^{2}$ with slope of $2.2 \times 10^{-9} \mathrm{~m}^{2} / \mathrm{s}$ as shown in Fig. 2a. The linear behavior indicates the Gaussian nature of the free diffusion of the water molecules within the sample. Note that the diffusion does not show restricted behavior since the single pore (grain) diameter is $300 \mu \mathrm{m}$ and the pore structure is not sampled by diffusion $\left(\langle Z\rangle=\sqrt{2 D_{m} \Delta} \ll 300 \mu \mathrm{m}\right)$ in time $\Delta$. This is true for all displacement observation times $\Delta$ used in this study. As Pe increases biexponential decay is observed, with a rapid decay region at low- $q$ followed by a transition to a slower decay rate of the same slope as the $P e=0$ data. The rapid spin echo signal decay at low- $q$ is due to larger displacements along the backbone flow generating increased signal dephasing. Measurement of the dynamics at varying displacement observation times $\Delta$ is shown in Fig. 2b) for the $P e=659$ flow rate. Linear fitting of the low $-q$ region slope provides the dispersion coefficient $K$ and hence the time dependent mean squared displacement $\left\langle Z^{2}(\Delta)>[19,21,32]\right.$.

The mean squared displacement data shown in Fig. 3) indicate a superdiffusive behavior of the mean squared displacement in
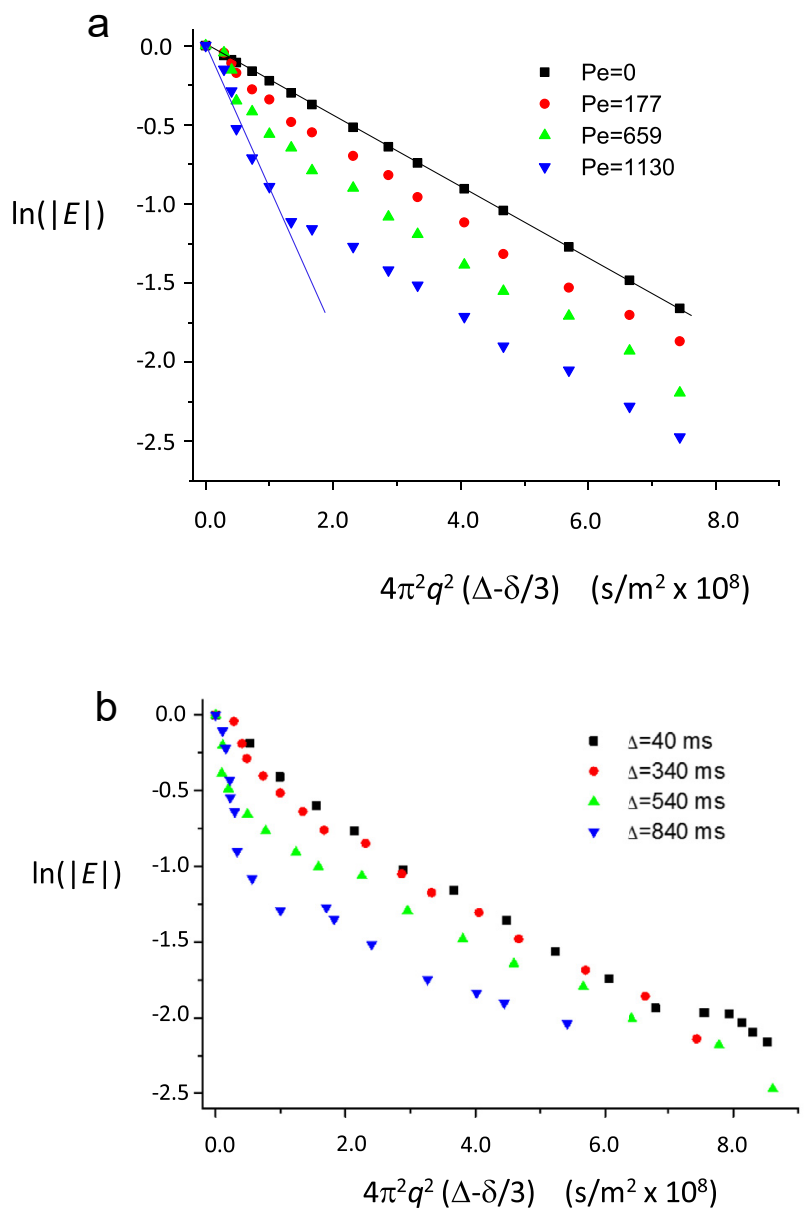

Fig. 2. Stejskal-Tanner plots used to calculate the low $q$ effective diffusion, or dispersion coefficient. a) Natural log of the echo attenuation function for varying Peclet number at fixed observation time of $\Delta=340 \mathrm{~ms}$. The initial decay proportional to the dispersion coefficient increases with increasing Pe. Note the Gaussian behavior at $P e=0$ is due to the fact that the percolation structure is not sampled by molecular diffusion $\left(D_{\mathrm{m}}=2.2 \times 10^{-9} \mathrm{~m}^{2} \mathrm{~s}^{-1}\right)$, i.e. $<\mathrm{Z}^{2}>^{1 / 2}=\left(2 D_{\mathrm{m}} \Delta\right)^{1 / 2} \ll 300 \mu \mathrm{m}$. The non Gaussian behavior increases under flow conditions with increasing time as does the initial decay proportional to the dispersion coefficient. b) Natural log of the echo attenuation function for hydrodynamic dispersion at varying displacement observation times $\Delta$ at $P e=659$.

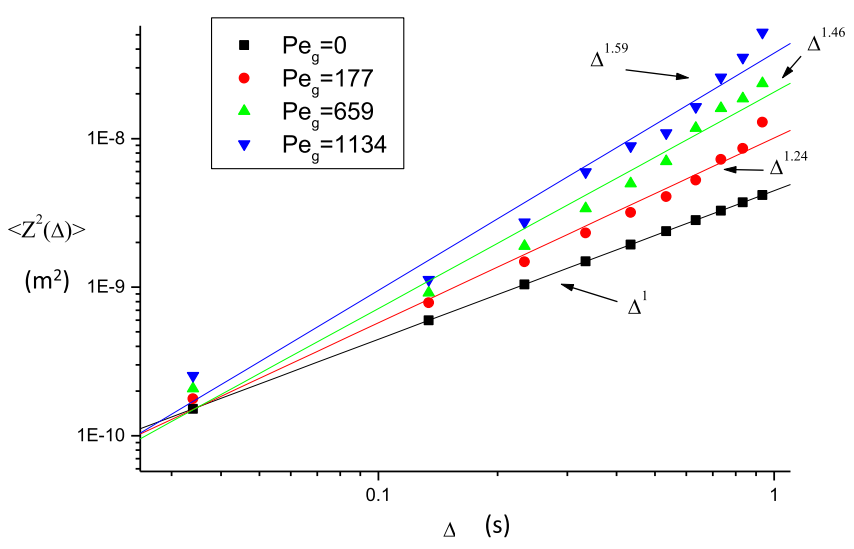

Fig. 3. The displacement time dependent variance for four values of $P e$. The dispersion is clearly superdiffusive with increasing exponent $\beta$ as Pe increases. The quality of the linear fits decreases as the flow rate increases in the accessible time observation window. The linear fit correlation coefficient is $\mathrm{R}^{2}=0.986$ at $P e=1134$. 
which the time scaling increases dependent on Pe. This result is in agreement with the simulations of Makse et al. but is in contrast to the classical site percolation model prediction of a fixed scaling dependent only on the geometry for a particular regime of $\mathrm{Pe}[9,12]$. As discussed above the simulations of Makse et al. predict a scaling for $P e>1.4$ of $\left\langle Z^{2}(\Delta)>\sim \Delta^{2 / d \text { min }}=\Delta^{1.76}\right.$ for the site percolation system studied here [12]. The data indicate a transition toward this theoretical maximum scaling as a function of $P e$, however the time dependent scaling is lower than this maxima even at $P e>1000$. A physical explanation for why the experimental data from these experiments do not completely comply with power laws observed at large Peclet numbers, may be that the object is not very much larger than the correlation length of the cluster, so that the statistics of averaging was unavoidably moderate.

\section{Conclusion}

Irrespective of how well percolation clusters are able to represent random porous media of practical relevance, their academic virtue is that structure/transport properties can be related to each other via scaling laws and characteristic exponents. That is, a small set of parameters defines and links both structure and transport features, at least qualitatively. In this way, the percolation concept forms a reference basis for porous media in general. The PGSE NMR data presented here for a $2 \mathrm{D}$ site percolation structure indicate the mean squared displacement due to hydrodynamic dispersion is superdiffusive. This result is consistent with classic percolation scaling concepts. However the time scaling of the mean squared displacement is dependent on the $P e$, in contrast to a constant value predicted by classic percolation theory based on percolation structure scalings.

\section{Acknowledgements}

JDS and SLC thank the Alexander von Humboldt Foundation for fellowship support during which the experimental work was done at the Universität Ulm and NSF CTS-0348076 (JDS) and CBET-
0642328 (SLC). The authors thank Dr. A. Klemm for sample construction and Mr. H. Wiringer and Dr. M. Weber for technical assistance.

\section{References}

[1] J.-P. Bouchaud, A. Georges, Phys. Rep. 195 (1990) 127.

[2] R. Metzler, J. Klafter, Phys. Rep. 339 (2000) 1.

[3] H. Scher, M.F. Shlesinger, J.T. Bendler, Phys. Today January 26 (1991).

[4] Y. Gefen, A. Aharony, S. Alexander, Phys. Rev. Lett. 50 (1983) 77.

[5] B. Berkowitz, H. Scher, Phys. Rev. Lett. 79 (1997) 4038.

[6] T. Geisel, J. Nierwetberg, A. Zacherl, Phys. Rev. Lett. 54 (1985) 616.

[7] J.-P. Bouchaud, et al., Phys. Rev. Lett. 64 (1990) 2503.

[8] M.F. Shlesinger, B.J. West, J. Klafter, Phys. Rev. Lett. 58 (1987) 1100.

[9] M. Sahimi, Flow and Transport in Porous Media and Fractured Rock: from Classical Methods to Modern Approaches, VCH, Weinheim, 1995.

[10] D. Lairez, et al., Phys. Rev. Lett. 98 (2007) 228302.

[11] P.-G. de Gennes, J. Fluid Mech. 136 (1983) 189.

[12] H.A. Makse, J.S. Andrade Jr., H.E. Stanley, Phys. Rev. E 61 (2000) 583.

[13] A.D. Stroock, et al., Science 295 (2002) 647.

[14] G.I. Taylor, Proc. R. Soc. Lond. Ser. A Math. Phys. Sci. 219 (1953) 186.

[15] R.F. Probstein, Physicochemical Hydrodynamics, Butterworth -Heinemann, Stoneham, MA, 1989.

[16] H. Brenner, J. Stat. Phys. 62 (1991) 1095.

[17] C. van den Broeck, Phys. A Stat. Theor. Phys. (Amst) 168 (1990) 677.

[18] D.L. Koch, J.F. Brady, Phys. Fluids 31 (1988) 965.

[19] S.L. Codd, et al., Phys. Rev. E 60 (1999) R3491.

[20] F. Klammler, R. Kimmich, Croat. Chem. Acta 65 (1992) 455.

[21] J.D. Seymour, P.T. Callaghan, AIChE J. 43 (1997) 2096.

[22] S. Stapf, et al., Phys. Rev. E 58 (1998) 6206.

[23] B. Manz, P. Alexander, L.F. Gladden, Phys. Fluids 11 (1999) 259.

[24] A. Klemm, H.-P. Muller, R. Kimmich, Phys. Rev. E 55 (1997) 4413.

[25] A. Klemm, R. Kimmich, M. Weber, Phys. Rev. E 63 (2001) 041514.

[26] A. Klemm, R. Metzler, R. Kimmich, Phys. Rev. E 65 (2002) 021112.

[27] R. Kimmich, Chem. Phys. 284 (2002) 253.

[28] Y. Li, G. Farrher, R. Kimmich, Phys. Rev. E 74 (2006) 066309.

[29] J. Koplik, S. Redner, D. Wilkinson, Phys. Rev. A 37 (1988) 2619.

[30] H.J. Herrmann, H.E. Stanley, J. Phys. A Math. Gen. 21 (1988) L829.

[31] E.O. Stejskal, J.E. Tanner, J. Chem. Phys. 42 (1965) 288.

[32] P.T. Callaghan, S.L. Codd, J.D. Seymour, Concepts Magn. Reson. 11 (1999) 181.

[33] R. Kimmich, NMR Tomography, Diffusometry, Relaxometry, Springer-Verlag, Berlin, 1997.

[34] J. Stepisnik, Phys. B Condens. Mat. (Amst) 183 (1993) 343.

[35] P.T. Callaghan, Principles of Nuclear Magnetic Resonance Microscopy, Oxford University Press, New York, 1991. 